\title{
Quality of Service Provisioning of Home eNodeB in OFDMA-based Wireless Network
}

\author{
[ Soon-Fatt Boo, Wai-Leong Pang, Sew-Kin Wong ]
}

\begin{abstract}
Femtocell is a small base station designed to increase the wireless network capacity and extend the coverage. It is a promising solution to overcome the tremendous growth in mobile traffic and indoor coverage problem. In this paper, extensive simulations have been carried out to evaluate the performance of the femtocell in an urban scenario, which compromised of indoor and outdoor users. The simulations are modelled in a heterogeneous network which consists of macrocell and femtocells. Femtocells are selectively deployed in an indoor environment. Proportional Fairness (PF), Modified Largest Weight Delay First (MLWDF) and Exponential Rule (ER) are used as the downlink scheduling algorithms. Simulation results show that femtocells significantly improved the network total throughput $(\sim 1200 \%)$ compared to the macrocell only scenario. Packet delay and packet loss ratio of video flows are reduced by $70 \%$. Meanwhile, fairness index of video flows are also improved by up to $50 \%$.
\end{abstract}

Keywords - OFDMA, Femtocell, Scheduling Algorithms, Quality of Service (QoS)

\section{Introduction}

Orthogonal Frequency Division Multiple Access (OFDMA) is introduced for downlink in LTE in order to improve the efficiency of the network in term of data rates, user diversity and frequency fading resistance [1]. OFDMA is an Orthogonal Frequency Division Multiple (OFDM) based multiple access technique that used in downlink transmission. It is also known as the combination of FDMA and TDMA techniques. The system bandwidth is divided into cluster of sub-carriers. The bandwidth is shared among multiple users simultaneously instead of providing whole bandwidth to a single user at a time.

The enormous growth of mobile network usage has becomes a tough challenge for service providers. Survey showed that more than $80 \%$ of the data traffics are generated from indoor environment [2].

Soon Fatt Boo

Multimedia University

Malaysia

Wai Leong Pang

Multimedia University

Malaysia

Sew Kin Wong

Multimedia University Malaysia
The eNodeB (eNB) is difficult to meet the indoor users demand because LTE network that operates at highfrequency band (for an example, $2.6 \mathrm{GHz}$ ) is tougher to penetrate through building walls. Therefore, indoor users will experience low Signal to Interference plus Noise Ratio (SINR) due to the high penetration loss. Femtocell also known as Home eNodeB (HeNB) was introduced in 3GPP release 8 in order to off-load the traffics in eNB and improve the indoor coverage. It is a small and low-power device that serves as a network access point for a small geographical area $(10-30 \mathrm{~m})$ and it operates in licensed spectrum. It connects the service provider's core network using the existing internet backhaul such as cable, digital subscriber line or optical fibers. HeNB is usually deployed in indoor (for example, home and business) or outdoor with limited coverage. The aim of this paper is to investigate the efficiency of HeNBs by comparing the scenario with the deployment of HeNBs and the scenario without the deployment of HeNBs in an indoor environment for mixed traffic flows.

This paper is organized as follow. Section II describes the downlink system model of LTE network. Section III discusses the simulation framework, i.e. simulation topology, traffic models, channel structure and resource management. Extensive simulation results are presented in section IV. Finally, a conclusion is drawn in section V.

\section{Downlink System Model}

Network architecture of LTE is simplified, where eNB is responsible for entire radio resource management in order to increase the network efficiency. Femtocells or HeNBs may be deployed within the eNB coverage. The combination of macrocell and femtocells is known as Heterogeneous Network (HetNet). S1-interfaces are the communication link for eNB and HeNB through the Mobility Management Entity (MME) or HeNB gateway. The LTE HetNet architecture is illustrated in Figure 1.

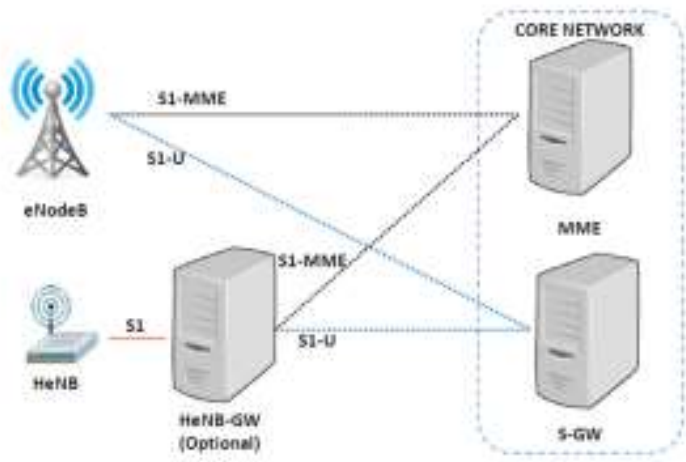

Figure 1. LTE HetNet Architecture 


\section{A. Physical Layer Structure}

In physical layer, the system bandwidth is divided into cluster of sub-carriers known as Resource Block (RB). It is the minimum unit allocated to the User Equipment (UE). RB is composed of one sub-channel of $180 \mathrm{kHz}$ (or equivalent to 12 sub-carriers of $15 \mathrm{kHz}$ ) in frequency-domain and one time slot of $0.5 \mathrm{~ms}$ which contains 7 OFDM symbols in time-domain. LTE is scalable in bandwidth which supports frequency bandwidth of $1.4,3,5,10,14$ and $20 \mathrm{MHz}$. The number of RBs are $6,15,25,50,75$ and 100 respectively [3]. The greater the bandwidth, the higher the number of RBs and hence more UEs can be supported simultaneously.

\section{B. Scheduling Algorithms}

Scheduling algorithm is responsible for resource allocation among variety of flows in wireless network. In this paper, three widely deployed scheduling algorithms, i.e. Proportional Fairness (PF), Modified Largest Weight Delay First (MLWDF) and Exponential Rule (ER) are used as downlink scheduling algorithms.

PF algorithm is developed to compromise the total data rate and fair data for each user. The channel quality experienced and past user throughput values are taken into account in order to fully utilize the network throughput and ensure the fairness among the flows. It is more suitable to apply on the non-real time flows [4].

$$
m_{i_{i} j}^{p F}=\frac{d_{j}^{i}(t)}{\bar{R}^{i}(t-1)}
$$

where $d_{j}^{i}(t)$ is the data rate requested, while $\bar{R}^{i}(t-1)$ is the average data rate of the UE $i$ at time slot $t$.

MLWDF algorithm takes instantaneous channel variations and delays into account in order to balance and schedule the delayed packets. Packets exceed the delay threshold value will be discarded. It is developed to support multiple real time applications with different QoS requirements [4].

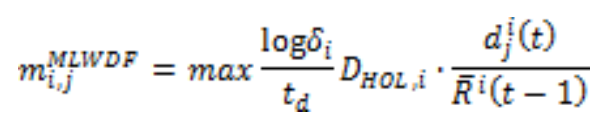

where $\delta_{\mathrm{i}}$ is the acceptable drop probability, $t_{d}$ is the target delay and $D_{\text {Hor } ;}$ is the Head of Line (HOL) packet delay.

ER algorithm is the modified version of PF algorithms with additional exponential term. Delay of the current user is normalized over the total delay of the past users. It is designed to support both real time and non-real time applications. The priority of real time flows is higher than non-real time flows [5].

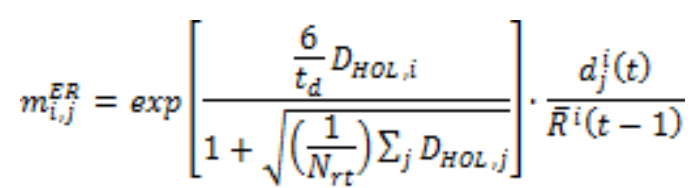

where $N_{r t}$ is the number of UEs with active real time flows (downlink).

\section{Framework Development}

Extensive simulations are carried out to evaluate the performance of the femtocells using LTE-Sim [6].

\section{A. Network Topology}

In this paper, a single hexagonal cell with radius of $1 \mathrm{~km}$ is developed. An eNB with 2 antenna ports is deployed at the center of the cell. A building with 25 apartment suites is also modeled. The size of each apartment suite is $100 \mathrm{~m}^{2}$. Two scenarios are developed, where apartment suites are deployed with HeNBs and without HeNB (indoor users are served by eNB). In the scenario with HeNBs, each HeNB will serve 2 users simultaneously. The total number of users available in the simulation is 50 users. The number of users is increased in a step of 10 users, from 10 users to 50 users. There are $80 \%$ of indoors users, and $20 \%$ of outdoor users in the scenario to realize the real world network topology. Users are moving at the speed of $3 \mathrm{~km} / \mathrm{h}$ randomly within the apartment or cell.

Meanwhile, multiple flows (1 VoIP, 1 video and $1 \mathrm{BE}$ ) are assigned to each UE. For VoIP flow, $8.4 \mathrm{kbps}$ bit rate of G.729 voice application is used [6]. It is designed with an $\mathrm{ON}$ and OFF Markov chain. The period for ON mode is distributed exponentially within $3 \mathrm{~s}$. The period for OFF mode is a truncated exponential probability density function with an upper limit of $6.9 \mathrm{~s}$ and an average of $3 \mathrm{~s}$. Packets with the size of 20 bytes is transmitted in every $20 \mathrm{~ms}$ during the ON period and no packets will be transmitted during the OFF period [6]. For video flow, $242 \mathrm{kbps}$ bit rate of H.264 video applications is used. It is a trace based application that delivers data based on the realistic video trace files in [7]. For BE flow, infinite buffer application is used. It is a model that continuously has data to send without QoS guarantee. Data rate and delivery time are varies depending on the network condition.

\section{B. Channel Structure}

The channel structure is modeled based on 3GPP specification. In frequency domain, downlink system bandwidth is set as $10 \mathrm{MHz}$ or is equivalent to $50 \mathrm{RBs}$. Frequency Division Duplex (FDD) is used as the frame structure with the carrier frequency of $2 \mathrm{GHz}$. Meanwhile, $1 / 4$ frequency reuse technique is implemented. In other words, the system bandwidth is shared among the cluster of 4 cells. In time domain, the LTE frame that consists of 10 subframes is modeled. Transmission Time Interval (TTI) of each sub-frame is set to $1 \mathrm{~ms}$. Each TTI consists of 2 time slots of $0.5 \mathrm{~ms}$. The eNB and HeNBs are synchronized in frequency and time domains. The maximum transmission power for eNB and $\mathrm{HeNB}$ are $43 \mathrm{dBm}$ and $20 \mathrm{dBm}$ respectively.

Propagation losses model i.e. path loss, penetration loss, shadowing and fast fading is considered in order to imitate the real world channel condition. The path loss (PL) is expressed in equation (4).

$$
\mathrm{PL}=(128.1+37.6 \log d) \mathrm{dB}
$$

where $\mathrm{d}$ indicates the distance between the eNB or HeNB and UE in kilometer. 
Proc. of The Fifth Intl. Conf. On Advances in Computing, Electronics and Electrical Technology - CEET 2016 Copyright (C) Institute of Research Engineers and Doctors, USA .All rights reserved.

ISBN: 978-1-63248-087-3 doi: 10.15224/ 978-1-63248-087-3-12

Meanwhile, the penetration loss is set to $10 \mathrm{~dB}$ while shadowing is set to log-normal distribution with the mean value of 0 and standard deviation of $8 \mathrm{~dB}$ [8]. Rayleigh fading model is implemented to model the fast fading due to multipath at different user speeds [9]. In the scenario without HeNBs, propagation losses of the indoor users are higher compared to outdoor users due to the obstacles such as building walls. Thus, additional $20 \mathrm{~dB}$ of attenuation factor is considered for indoor users. The simulation parameters are summarized in Table 1.

Table 1. Simulation Parameters

\begin{tabular}{c|c}
\multicolumn{2}{c}{ Table 1. Simulation Parameters } \\
\hline \hline Parameter & Value \\
\hline Cluster Size & 4 \\
\hline Cell Radius & $1 \mathrm{~km}$ \\
\hline System Bandwidth & $10 \mathrm{MHz}$ \\
\hline Frame Structure & FDD \\
\hline TTI & $1 \mathrm{~ms}$ \\
\hline Maximum Transmission & eNB: $43 \mathrm{dBm}$ \\
Power & HeNB: $20 \mathrm{dBm}$ \\
\hline No. of UEs & $\{10,20,30,40,50\}$ \\
\hline Mobility Speed & $3 \mathrm{~km} / \mathrm{h}$ \\
\hline
\end{tabular}

\section{Simulation Results}

The duration of the simulation is $100 \mathrm{~s}$ and repeated for 5 times. Average results are taken in order to improve the precision and accuracy of the simulation results.

\section{A. Performance Metrics}

The performance metrics evaluated in this paper are throughput, packet delay, packet loss ratio and fairness index.

Throughput is the total data rates that successfully delivered to the users in time. Packet delay is the period of time for a packet to reach its destination successfully. Packets failed to reach its destination within the upper delay threshold will be discarded. Packet Loss Ratio (PLR) determines the ratio of the dropped packets over the total number of packets sent. Fairness index determines the equality of resource distribution among the users. This metric value is ranged from 0 (worst) to 1 (best).

\section{B. Results and Discussion}

The network performance in the scenario with the deployment of HeNB and without HeNB are evaluated and compared in this section. PL, MLWDF and ER are used as the downlink scheduling algorithms. Total system throughput is shown in Figure 2. Result shows that HeNBs significantly improved the network throughput. HeNBs provide approximately $1200.0 \%$ higher throughput as compared to the scenario without HeNBs regardless of the scheduling algorithms and the number of users. Indoor users attached to eNBs may suffer from high penetration losses due to the obstacles such as building walls.

The packet delay of VoIP and video are shown in Figure 3 and 4 respectively. The variations of VoIP delay between the scenario with HeNBs and without HeNBs are not significant. However, the delay of VoIP for PF without HeNBs is slightly higher at 20 and 50 users as compared to the others. Meanwhile, the delay of the video for MLWDF with HeNBs is slightly higher compared to PF and MLWDF without HeNBs at 10 users. However, the former able to maintain a low delay when the number of users increases up to 50. The difference between PF with HeNBs and PF without HeNBs is most significant, which improved the video delay by $70.5 \%$ at 50 users.

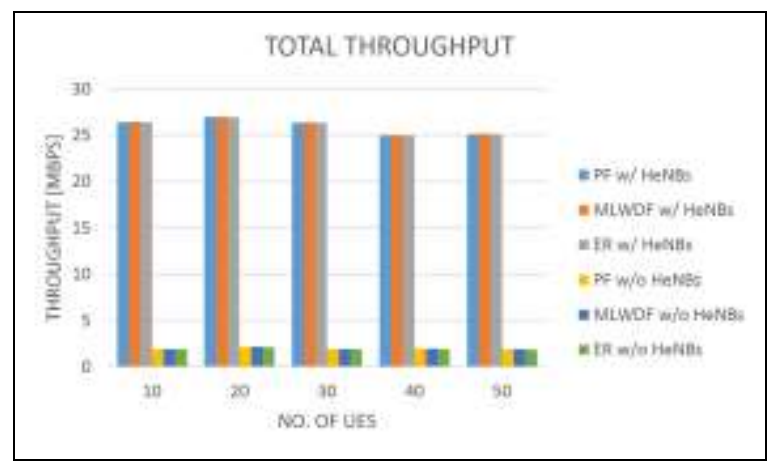

Figure 2. Total Throughput

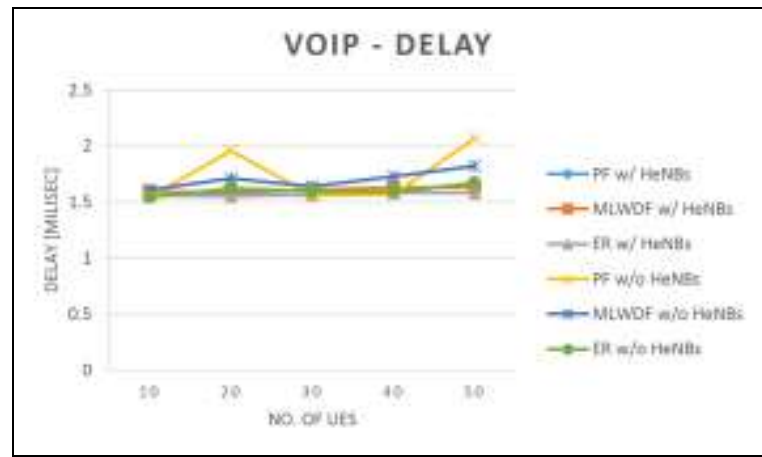

Figure 3. Delay of VoIP

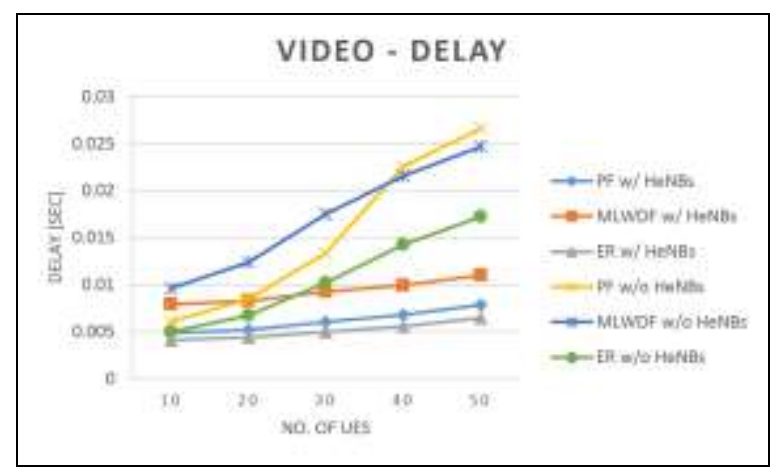

Figure 4. Delay of video 
Proc. of The Fifth Intl. Conf. On Advances in Computing, Electronics and Electrical Technology - CEET 2016 Copyright (C) Institute of Research Engineers and Doctors, USA .All rights reserved.

ISBN: 978-1-63248-087-3 doi: 10.15224/ 978-1-63248-087-3-12

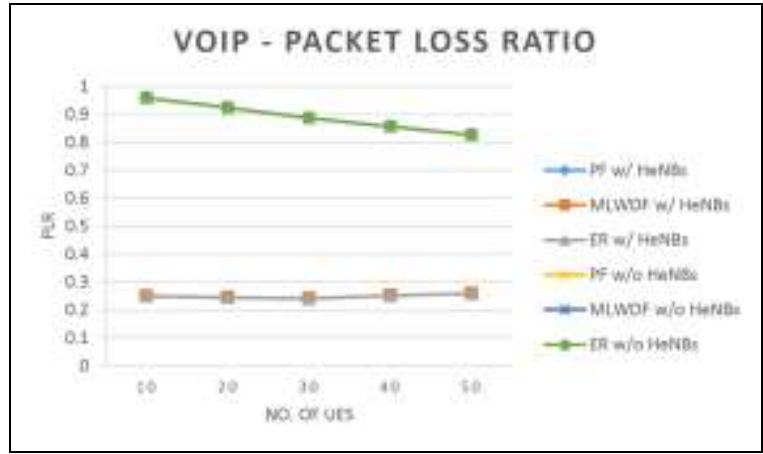

Figure 5. Packet loss ratio of VoIP

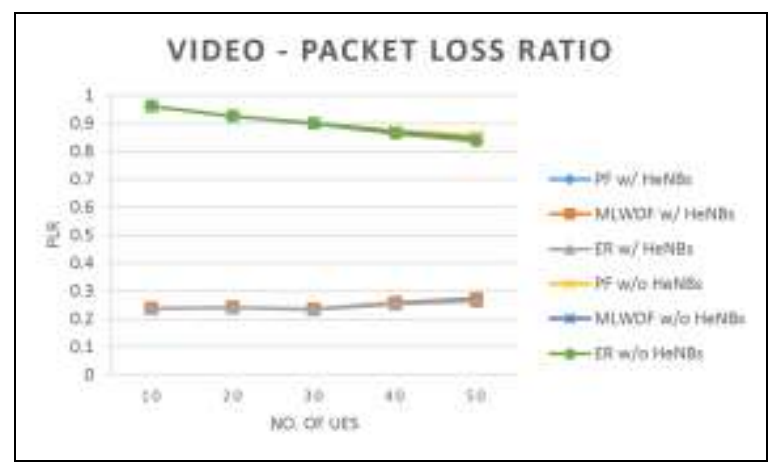

Figure 6. Packet loss ratio of video

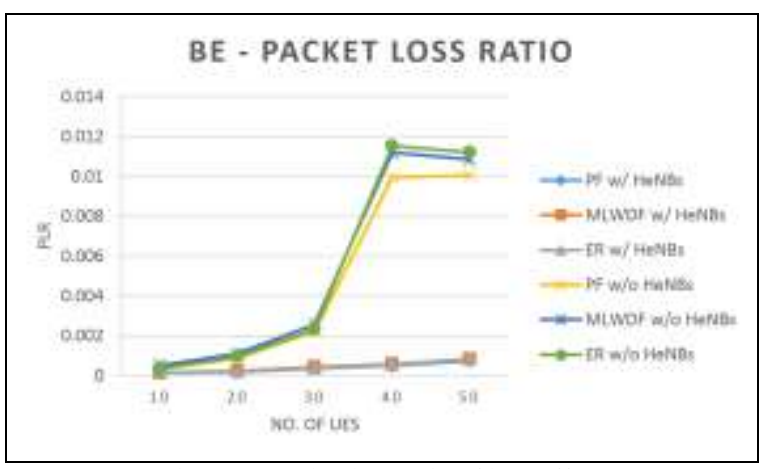

Figure 7. Packet loss ratio of best effort

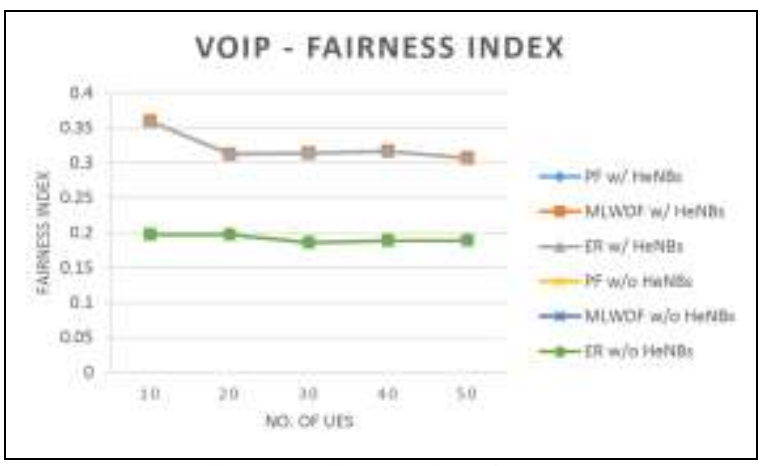

Figure 8. Fairness Index of VoIP

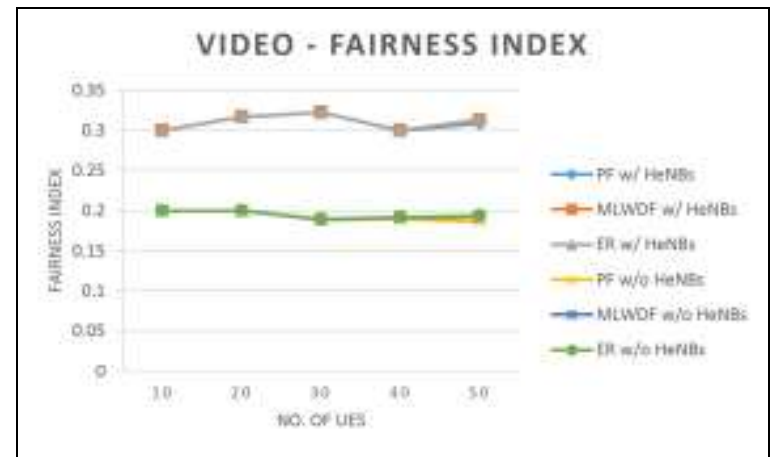

Figure 9. Fairness Index of video

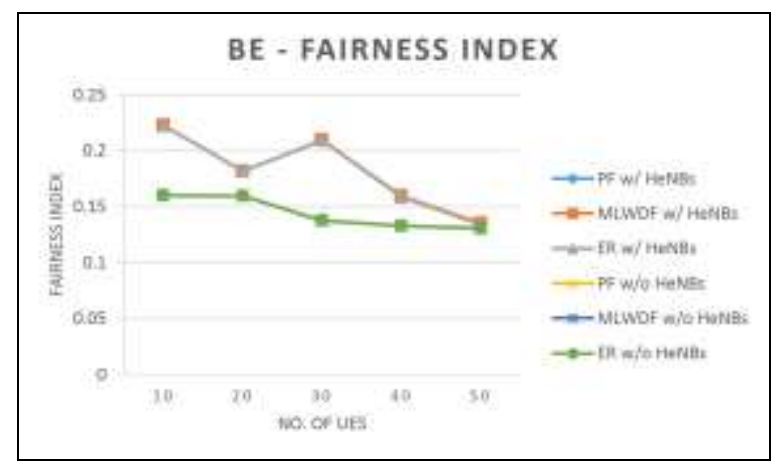

Figure 10. Fairness Index of best effort

The packet loss ratio of VoIP, video and BE are shown in Figure 5, 6 and 7 respectively. Packet loss ratio of VoIP and video are reduced about $68.0 \%-75.0 \%$ when HeNBs are deployed regardless of number of users. Meanwhile, the packet loss ratio of $\mathrm{BE}$ is decreased by $92.4 \%$ at 50 users.

The fairness index of VoIP, video and BE are shown in Figure 8, 9 and 10 respectively. The fairness index values of VoIP and video are increased about $50.0 \%$ when HeNBs are deployed at 50 users. Moreover, the fairness index of BE increased by $37.5 \%$ at 10 users but no improvement when the number of users increases up to 50 . The maximum fairness index are approximately 0.35 instead of 1 is due to the resources sharing among the three different flows in one UE.

In general, the results show that deployment of HeNBs is able to improve the overall performance of the network by off-loading traffics in eNB and improve the indoor coverage.

\section{v. Conclusion}

The objective of this paper is to evaluate the performance of HeNBs in an indoor environment using three different downlink scheduling algorithms i.e PF, MLWDF and ER. Extensive simulation results show that the deployment of HeNB can effectively improves the performance of the network in term of throughput, delay, fairness index and packet loss ratio. This shown that the HeNB deployment is a promising solution that significantly improves the network performance and enhances the QoS provisioning to the real time and non-real time flow. Further work may consider on the interference between the HeNBs. 


\section{References}

[1] M. Iturralde, T. A. Yahiya, A. Wei, and A.L. Beylot, "Performance Study of Multimedia Services Using Virtual Token Mechanism for Resource Allocation in LTE Networks," IEEE Vehicular Technology Conference, 1-5, Sept 2011.

[2] K. Fitchard, "Report: $80 \%$ of All Mobile Data is Consumed by just $10 \%$ of Users," _ https://gigaom.com/2015/02/04/report-80-of-allmobile-data-is-consumed-by-just-10-of-users, 2015.

[3] 3GPP, Tech. Specif. 3GPP TS 36.322. Group Radio Access Network Evolved Universal Terrestrial Radio Access (E-URA), Radio Link Control (RLC) protocol specification (Release 9).

[4] G. Piro, L.A. Grieco, G.Boggia, F.Capozzi, and P. Camrda, "Downlink Packet Scheduling in LTE Cellular Networks: Key Design Issues and a Survey," IEEE Communication Surveys and Tutorial, vol. 15, issue 2, pp. 678-700, May 2013.

[5] S. Dardouri, and R. Bouallegue, "Comparative Study of Downlink Packet Scheduling for LTE Networks," Wireless Pers. Comm., vol. 83, issue 3, pp. 1405-1418, Jan 2015.

[6] G. Piro, L.A. Grieco, G.Boggia, F.Capozzi, and P. Camrda, “ Simulating LTE Cellular Systems: an Open Source Framework", IEEE Transaction Vehicular Technology," vol. 60, issue 2, pp. 498513, Feb 2011.

[7] Video Trace Library. http://trace.eas.asu.edu/

[8] 3GPP, Tech. Specif. 3GPP TS 25.814. Group Radio Access Network Physical layer aspect for evolved Universal Terrestrial Radio Access (UTRA) (Release 7).

[9] 3GPP, Tech. Specif. 3GPP TS 25.942. Group Radio Access Network Radio Frequency (RF) system scenarios (Release 9).

About Author (s):

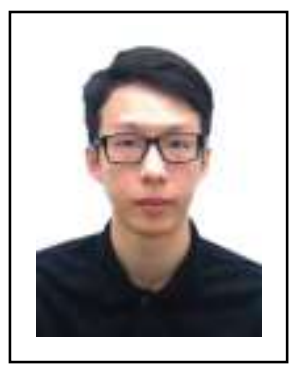

Soon-Fatt Boo received B.E degree in Electronics majoring in Telecommunication from Multimedia University, Malaysia in March 2015. Currently a M.Eng.Sc student at Multimedia University, Malaysia. His research interests focus on the media access control, scheduling algorithms and quality of service in the OFDMA-based wireless network.

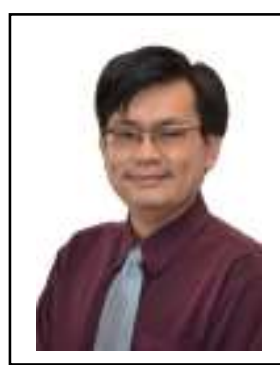

Wai-Leong Pang received B.E. degree in Electrical and Electronics in 2001, Master of Science (Microelectronics) in 2003 and the Ph.D. degree (Communications and Networking) in 2013. Since 2004, he has been with Multimedia University, Malaysia. His research interests include wireless communications, networking, VLSI, digital design and renewable energy.

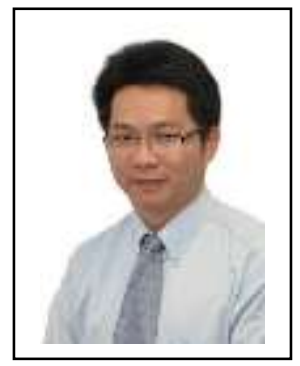

Sew-Kin Wong received his B.Eng degree in Electrical and Electronic Engineering in 1995. He then obtained his M.Eng.Sc degree and Ph.D degree in 2003 and 2011 respectively. His research interests are RF transceiver and RFIC design for the next generation wireless systems and EMI/EMC compliances and shielding techniques. He is currently a member of the Faculty of Engineering, Multimedia University, Malaysia. 